\title{
Applying QoS in MANET using Stigmergy of Ants
}

\author{
Sanjeev Kumar \\ Department of Information \\ Technology \\ ABES Engineering College, \\ Ghaziabad
}

\author{
Santosh Kr. Paul \\ Department of \\ Computer Science \\ ABES Engineering College, \\ Ghaziabad
}

\author{
Shyam Singh Rajput \\ Department of \\ Computer Science \\ ABES Engineering College \\ Ghaziabad
}

\begin{abstract}
As current research is focusing on implementation of MANET for various commercial applications. But providing quality of service in Mobile Ad Hoc Networks is not an easy task due to the resource limitations and dynamic nature of MANETs. This paper presents a proposal for maintaining quality of service parameter for communication in MANET. This proposal is mainly inspired by the concept of swarm intelligence exhibited by group of ants when they search for food. Diffserv like service classes can be made available through this proposed approach. The approach has inherited advantage of robustness and fast convergence from swarm intelligent system, which makes it a better choice over existing QoS algorithms for MANET.
\end{abstract}

\section{Keywords}

Swarm intelligence, Ant colony optimization, Mobile Ad Hoc networks, Quality of Service

\section{INTRODUCTION}

Mobile ad hoc network, a multi hop mobile radio network [1] is a self-organizing and rapidly deployable network in which neither a wired backbone nor a centralized control exists. As It is known that MANET exhibits special property that it is mobile in nature so the node mobility can change the topology and hence the path once setup may be best one but not for long time. The network nodes communicate with one another over scarce wireless channels in a multi- hop fashion. The ad hoc network is adaptable to the highly dynamic topology resulted from the mobility of network nodes and the changing propagation conditions. The rising popularity and commercial usage of MANETs demands support for quality of service is such networks to work effectively.

A lot of research is being carried out in the area of providing quality of service in mobile ad hoc networks but work is still in its infancy stage [5].

This paper proposes an approach inspired from the emergent behavior in social insects. Recent research in swarm intelligence has revealed matching properties between the routing requirements of communication networks and certain tasks that exist in social insect colonies [2].

The approach tries to keep the process simple and therefore goes for a hybrid approach. Path to a destination is only established if demanded by any application. While this path is in use by the application, proactive forward ants move in the network to explore and collect other routes that may act as alternatives. There has been used multiple ways to keep routing table updated and for this purpose, It is always tried to use a process that produces acceptable overhead. Pheromone evaporation and pheromone diffusion phenomenon are used to keep the routing tables short as well as replicate useful routes throughout the network quickly.

\section{QUALITY OF SERVICE}

The quality of service is defined as the collective effect of service in which the network is expected to guarantee a set of measurable pre- specified service attributes to users in terms of end to end performance, such as delay, bandwidth, probability of packet loss and jitter.

In wire based network the quality of service can be provided in two ways i.e., over-provisioning of resources and network traffic Engineering.

Through over-provisioning of resources adds plenty of resources but all the users remain at one service class. In network traffic engineering the users are divided in classes according to their application requirements and each class is given defined priority.

Network traffic engineering has two main types named reservation-based engineering and reservation-less engineering. In reservation- based engineering, network resources are reserved for particular application. Integrated service (Intserv) [3] is the method of reservation based engineering having RSVP an out of band signaling protocol. The differentiated service (Diffserv) [4] is the main approach in reservation-less engineering.

\subsection{Challenges for providing QoS}

The Inherent nature of MANET imposes variety of issues with regard to QoS parameters, which include wireless medium, decentralization, broadcast nature, mobility, power consumption and complex routing etc. Further there are partial and over reservations which are specifically related to QoS, are witnessed in MANETs, which again contribute to the above mentioned challenges. The main Internet quality of service approaches, Intserv and Diffserv, cannot be directly applied in MANETs because Intserv is state full approach requiring huge processing and storing at intermediate nodes to keep state information, which also turns into a scalability issue. The RSVP, which is the main ingredient of Intserv, is network consuming process, which is fatal for already bandwidth limited MANET. Diffserv on the other hand is a light weight approach for MANET but due to dynamic topology in MANET, router definitions as ingress, core, and egress are difficult. Even the concept of service level agreement is difficult to apply in MANET.

\section{ROUTING}

Keeping in view the resource limitation and highly dynamic topology, a lot of research has been conducted in the routing area of MANETs and a plethora of routing protocols exists for MANETs [5].

On the basis of routing strategy used, these protocols can be classified as Proactive, reactive and a combination of theses two called hybrid protocols. Proactive routing protocols are the same old traditional wired LAN/WAN protocols tailored towards mobile ad hoc networks. These protocols consume larger bandwidth for frequent updates in a highly dynamic 
topological network like MANET. Since the bandwidth is at premium in wireless network and hence its usage must be minimized, therefore a proactive routing is not the best candidate for a routing algorithm in MANETs. Some of the proposed proactive protocols are DSDV, WRP and OLSR.

On the other hand reactive routing protocols avoid larger frequent updates by initiating route discovery only when needed. This minimizes the bandwidth usage but offers delay when a packet needs a transmission as the global search is conducted before actual transmission. Some of the proposed reactive routing protocols are AODV, DSR and TORA.

The third type of proposed routing protocols is the combination of both, the proactive and reactive routing protocols, and hence is called hybrid routing protocol. These protocols attempts to collect advantages of both the approaches and can produce acceptable results in most of the situations whereas the previously said approaches usually depend upon the environment for optimal performance. A popular example of hybrid protocols is ZRP.

\subsection{QoS aware Routing}

None of the above mentioned protocols take into account the Quality of Service rather these are only best effort delivery protocols in nature. Where QoS is a set of service requirements that needs to be met by the network while transporting a packet stream from a source to its destination, therefore, the primary goal of the QoS-aware routing protocols is to determine a path from a source to the destination that satisfies the needs of such desired QoS level. The nature of MANET does not guarantee such an assurance. Hence providing quality of service aware routing in MANETs is a more complex issue.

QoS-aware routing is a growing research area in MANETs and some schemes have been proposed involving extensions to the already available best effort routing protocols to achieve certain amount of quality of service.

Perkins et. al. [6] extends reactive routing protocols in such a way that the Route Request packets also carry along with themselves the desired QoS metrics. This approach not just makes the packet header large enough in case of basic reactive protocols but also generates huge amount of traffic because of flooding of those packets. The large sized route request also adds into latency.

Kunz et. al. [7] modified proactive protocols such that their routing tables also contain the link status in terms of quality of service metrics. The mobility issue of MANETs discourages the use of pure Proactive protocols in most environments, while the QoS metrics, by utilizing extra bandwidth for maintaining the routing tables, further makes them hard to operate effectively.

\subsection{Swarm Intelligence Based Routing}

Recently, a new technique emerged for solving optimization problems which is inspired from emergent swarm intelligence in behavior of social insect colonies [7]. Usually thousands of small agents like ants with very little direct communication solve global optimization problems like shortest path to a food source. Ants use a chemical substance called pheromone for indirect communication between them. Pheromone concentration increases as more ants traverse the same path and that attracts other ants too, hence convergence appears. Diffusion property of this pheromone is an important one for mobile networks. The process of indirect communication between agents by modification induced in environment through this pheromone is known as stigmergy [9]. Research in swarm intelligence has revealed great matching properties between this stigmergy and communication networks routing. Some algorithms have been proposed for wired networks. Most popular of these is Antnet proposed by Gianni Di Caro ad Marco Dorigo [2]. The basic difference between swarm intelligence routing and traditional routing algorithms is that the former uses stochastic exploration of routes depending upon the pheromone concentration. In a simple routing, ants are used as mobile agents for route exploration much like ant foragers in real ant colonies. There are usually two types of ants namely forward and backward ants. Forward ants are sent for exploration and while reaching at destinations these ants die out after transferring acquired network information to backward ants. These ants in turn are usually routed back to source while updating the routing tables at intermediate nodes. In this process, routing tables contain updated pheromone values as a measure of good put of using the corresponding path.

Swarm intelligence based routing protocols are robust, dynamic and rely on local information only. Furthermore, multiple routes are also achieved through ant colony based algorithms, which are also the desired properties of MANET routing protocols. Some routing protocols based on swarm intelligence have been proposed for MANETs, which have proven results for lesser overhead and more efficiency $[9,10,11]$

\section{PROPOSED ALGORITHM}

In this section it is proposed that a QoS aware routing algorithm based on stigmergy found in ant colony, which is a process of indirect communication through modifications induced in environment between agents.

The proposed algorithm is hybrid in nature. Part of it is on demand as the route exploration is only carried out when required so that unnecessary control traffic is minimized. However, once data path is established, other paths to the same destinations are explored so that in case of link failure, data could be forwarded in acceptable interruption. This is greatly helpful in case of QoS applications. This part of approach imitates the idea from proactive algorithms. The algorithm assumes bidirectional symmetric links all the time.

In this approach, there are two types of agents i.e., forward ants and backward ants. The forward ants have two responsibilities. First they are used to find out a path to a destination fulfilling the QoS requirements, whenever there is request for data transfer pending for that. For this purpose, source produces a forward ant. It is usually broadcast in this regard but may be unicast in its way if an entry for the required destination is found in the routing table of any intermediate node that matches to the $\mathrm{QoS}$ metrics involving minimum required bandwidth and maximum permissible delay. Along with these metrics, forward ants also carry the source and destination addresses, sequence number and a stack on which they keep the information about all the intermediate nodes.

Once ant gets to the destination, it dies out to give birth to a backward ant, which is unicast and directed back to the source on the path, discovered by the forward ant. On its return, it modifies the routing tables of all the intermediate nodes with the new experience, gathered by the forward ant. When the backward ant gets to the source, a path is established and data transfer starts.

While applications are communicating, the proactive part of the algorithm activates. It sends the forward ants randomly on 
relatively idle paths to explore for possibly better alternate routes to the current destination. This is helpful in two respects: first in case of topology change (as with the nature of MANETs) it will have multiple options for the application, thus keeping operating without interruption; secondly, in case of high QoS requirements of the application, which it is hard for a single path to fulfill, load balancing could be applied to facilitate the operation.

\subsection{Pheromone Diffusion}

Di Caro in [10] gave impressive concept of pheromone diffusion. This is of great help in our approach too. According to their approach, hello messages (that neighbor's send to each other for ensuring their presence) contain information about active destinations. The active destinations are defined to be those nodes with which currently communication had taken place. In this way, an active destination is introduced to all the neighbors and this information propagates through the network creating and maintaining entries in the routing tables without extra effort.

\subsection{Pheromone Evaporation}

Swarm intelligence has built-in property of negative reinforcement i.e., whenever a backward ant will return and deploy results to the nodes, routing tables on those nodes will be computed not just considering the experience of backward ant but also the previous value of that route if it is not a new entry in the routing table. Thus the backward ant will also update a path that has lost quality. Moreover, it will use pheromone evaporation phenomenon of ant colony according to which goodness of a route present in the routing table decreases with time without even checking that route. So in this way it will remove those entries from the routing table which are not in use by any application thereby, keeping the routing table as short as possible.

The routing tables are kept updating through different techniques including (i) backward ants which is the core source of creating and maintaining routing tables, (ii) through pheromone diffusion and (iii) through the volatility of the pheromone which is not associated to any check but just depending upon time and usage of a path.

\subsection{Routing table structure}

The structure of the routing table maintained by every node is similar to Liliana's structure . For each destination i, the probability of $\mathrm{j}$ to be selected as next hop depends upon a function of bandwidth and delay associated with that link so it can be written as: $\mathrm{PQoS}-\mathrm{ij}=\mathrm{F}($ (PD-ij PB-ij) For any destination $i$, the sum of probabilities of all the network node that could be used as next hop will be exactly equal to one i.e., i jN H PQos-ij = 1 where $\mathrm{j}$ is next hop and $\mathrm{N}$ is neighbors.

\section{A SAMPLE NETWORK EXAMPLE}

Let us consider figure 1 . It is a simple network used for discussion about the approach under consideration. Node ' $S$ ' needs a route to destination ' $\mathrm{D}$ ' for a class of service having probability value 0.3 or above. The forward ant is sent and considering it returns the path through ' $\mathrm{J} 2$ ', which is capable to support desired service class so the path is forwarded to application and communication starts.

In the second phase, proactive forward ants come into action and attempts to find alternative paths to the same destination. Path through 'J3' is rejected because it could not only provide required QoS service but the path through ' $\mathrm{J} 1$ ' is selected as an alternative. If the path through ' $\mathrm{J} 2$ ' fails to meet the QoS requirements then the application could shift to the secondary path as well as it could use both the path for load balancing in case if both the paths fail to fulfill the requirements of the application

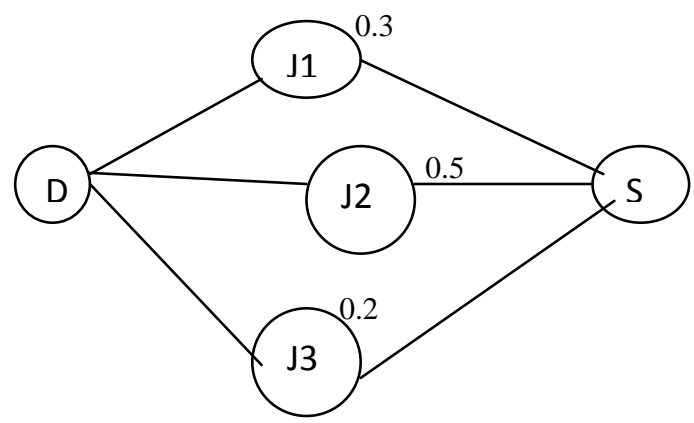

Figure 1: A Sample Network.

The goodness of a path is calculated based on a function of additive delay of the route and the minimum/bottleneck bandwidth available. This relation may be modified according to the environments and requirements. Note that outgoing path that could route a packet to destination ' $D$ ' are given values in such a way so that sum of probability values of all those routes always create one.

\section{CONCLUSION}

A QoS aware routing algorithm has been proposed inspired from an emergent area of swarm intelligence which is found in nature like bird flocking and ant colony etc. Simple ant agents' contribution in complex stigmergic process gives rise to larger optimization solutions. This technique when applied in communication networks can present self- regulatory routing patterns.

The Proposed approach attempts in reducing the routing overhead. No timely updates are used for updating routing table rather pheromone evaporation attempt to remove any entries that may be alive just to keep the routing table short. On the other hand, while communication is in progress, other routes to the same destination are searched in order to overcome the primary issue of MANETs i.e., mobility and link lost. Negative feedback in swarm intelligence is an important phenomenon for removing undesired route entries.

The hybrid quality of the algorithm makes it suitable for all the environments whereas reactive and proactive protocols are limited i.e., reactive will work best when the devices are mobile while proactive assumes more stable environment.

The proposed algorithm, being based on swarm intelligence, is robust and generates acceptable control overhead while giving multiple feasible routes for multimedia traffic.

\section{REFERENCES}

[1] Internet Engineering Task Force MANET Working group. http://www.ietf.org/html.charters/manetcharter.html

[2] G. Di Caro and M. Dorigo, "AntNet: distributed stigmergic control for communications networks", Journal of Artificial Intelligence Research, vol. 9, pp. 317-365, 1998.

[3] R. Braden, D. Clark and S. Shenker, "Integrated Services in the Internet Architecture" (RFC 1633), network working group, June 1994. http://www.ietf.org/rfc/rfc1633.txt 
[4] M. G"unes, U. Sorges, and I. Bouazisi, "Ara - the antcolony based routing algorithm for manets," in Proceedings of the ICPP Workshop on Ad Hoc Networks. IEEE Computer Society Press, 2002

[5] S. Blake, D. Black, M. Carlson, E. Davis, Z. Wang, W. Weiss, "An Architecture for Differentiated Services" (RFC 2475), network working group, December 1998 http://www.ietf.org/rfc/rfc2475.txt

[6] Padmini Misra, "Routing Protocols for Ad Hoc Mobile Wireless Networks" Ohio state university 2000.

[7] C. E. Perkins, E. M. Royer, S. R. Das, "Quality of Service for Ad hoc On-Demand Distance Vector Routing,"
[8] Y. Ge, T. Kunz, L. Lamont, "Quality of Service Routing in Ad-Hoc Networks Using OLSR,"Proceedings of the 36th Hawaii International Conference on System Sciences (HICSS'03) by IEEE in January 2003

[9] Dorigo, M. and Di Caro, G., The Ant ColonyOptimization Meta-Heuristic, New Ideas in Optimization, McGraw-Hill, 11-32, 1999.

[10] G. Di Caro, F. Ducatelle, and L.M. Gambardella. AntHocNet: an adaptive nature-inspired algorithm for routing in mobile ad hoc networks. European Transactions on Telecommunications, 2005. 